\title{
PPI-GA: A Novel Clustering Algorithm to Identify Protein Complexes within Protein-Protein Interaction Networks Using Genetic Algorithm
}

\author{
Naeem Shirmohammady, Habib Izadkhah (D), and Ayaz Isazadeh \\ Department of Computer Science, Faculty of Mathematical Sciences, University of Tabriz, Tabriz, Iran \\ Correspondence should be addressed to Habib Izadkhah; izadkhah@tabrizu.ac.ir
}

Received 15 June 2020; Revised 12 January 2021; Accepted 10 March 2021; Published 25 March 2021

Academic Editor: Mojtaba Ahmadieh Khanesar

Copyright (C) 2021 Naeem Shirmohammady et al. This is an open access article distributed under the Creative Commons Attribution License, which permits unrestricted use, distribution, and reproduction in any medium, provided the original work is properly cited.

\begin{abstract}
Comprehensive analysis of proteins to evaluate their genetic diversity, study their differences, and respond to the tensions is the main subject of an interdisciplinary field of study called proteomics. The main objective of the proteomics is to detect and quantify proteins and study their post-translational modifications and interactions using protein chemistry, bioinformatics, and biology. Any disturbance in proteins interactive network can act as a source for biological disorders and various diseases such as Alzheimer and cancer. Most current computational methods for discovering protein complexes are usually based on specific topological characteristics of protein-protein networks (PPI). To identify the protein complexes, in this paper, we, first, present a new encoding method to represent solutions; we then propose a new clustering algorithm based on the genetic algorithm, named PPIGA, employing a new multiobjective quality function. The proposed algorithm is evaluated on two gold standard and real-world datasets. The result achieved demonstrates that the proposed algorithm can detect important protein complexes, and it provides more accurate results compared with state-of-the-art protein complex identification algorithms.
\end{abstract}

\section{Introduction}

Proteins are essential nutrients which carry out cellular activities. They are also vital to our understanding of molecular functions and biological processes. In biological organisms, proteins are typically organized into a protein complex. When two or more are in physical contact, due to electrostatic forces or chemical phenomena, protein-protein interaction occurs. Such interactions often lead to biological functions. Many important molecular processes within a cell such as DNA replication and even protein metabolism are performed by large molecular complexes which consisted of many organized proteins interacting with each other.

It is important to expose protein interactions because it improves our knowledge about diseases and can establish new therapeutic methods. Although there are many experimental and computational methods to detect protein complexes, all of them have their advantages and disadvantages. Experimental approaches like yeast two-hybrid screening and tandem affinity purification with mass spectrometry can be used to detect protein complexes [1]. There are many biophysical methods to investigate the nature and properties of these interactions. These methods have certain limitations which are presented in [2]. For example, PPI data extracted from experiments typically have high false-negative and false-positive rates which have a negative impact on the accuracy of the experiment results. Although various computational density-based approaches were proposed to find connected subgraphs in PPI networks over the past decade, there are still some challenges to effectively detect protein complexes based on main properties and topology of the network which reduces the accuracy of results. Theoretically, graph theory is usually used to model the interactions among artifacts (here proteins). Thus, the developed computational approaches are used as a supplement to experimental methods. To detect the protein complexes, the set of all the protein-protein interactions of a given organism is 
naturally modeled by an undirected graph $G(V, E)$ in which each vertex $V$ and each edge $E$ represent a protein and the interaction between two proteins, respectively.

Protein detection in an interactive protein network is challenging which depends on some factors. Extracted interactive data usually have high false-negative and falsepositive rates. Also, each protein can belong to multiple protein complexes. Computational methods for protein complex detection mostly focus on extracting clusters, i.e., complexes, from PPI networks [1]. The purpose of PPI networks clustering is to find a group of proteins that interact and participate in the same biological process or proteins that perform a particular biological function. Since detecting protein complexes is an NP-hard problem, evolutionary algorithms such as genetic algorithm are utilized to find near-optimal solutions [3].

This paper presents a graph clustering algorithm with a novel multiobjective quality function and a new compacted encoding method to identify protein complexes from PPI networks. In this approach, we employ the genetic algorithm to find clusters for protein complexes. Comparing our results to previous algorithms shows that the proposed algorithm outperforms the existing clustering algorithms. Our approach has the highest computational accuracy among the compared approaches. The algorithm can detect clusters with different densities and complexes. Our algorithm is scalable so that the algorithm can cluster a large network without any disruption to its performance. follows:

The contributions of the paper are summarized as

(1) Presenting a new multiobjective quality function aiming to identify protein complexes;

(2) Presenting a new encoding method to show the solutions in the evolutionary process of the genetic algorithm, aiming to reduce the search space. The solution space produced by existing evolutionary algorithms is $n^{n}$, where $n$ is the number of proteins. The encoding proposed in this paper reduces the state space up to $n$ ! which is much smaller in comparison to $n^{n}$. This reduction in the search space makes (1) solving the problem easier for the GA and (2) converging faster and finding acceptable solutions during earlier generations.

The rest of this paper is organized into five sections. Section 2 addresses available algorithms to predict protein complexes. Section 3 describes the proposed clustering algorithm. In this section, we will present a new encoding and a new multiobjective function. Section 4 compares and analyzes the empirical results of different algorithms using various evaluation criteria. Section 5 presents the conclusion and future work.

\section{Related Work}

Proteins are a vital component of living beings, and they play a key role in managerial and executing most biological processes. Many clustering algorithms have been designed to detect meaningful groups of proteins from PPI networks [4].
There are three main approaches to cluster graphs aiming protein complex detection. The first one is to find subgraphs with specified connections which are called network motifs and is described as a complex or a part of it. Clique is one of the subgraphs detected using this approach. Due to the time complexity of this approach, its application is limited nowadays. The second one is graph-growing in which a cluster grows and completes around a vertex as a seed using greedy search algorithms. The third one includes several variants. The algorithms try to minimize or maximize measures of a specified cluster such as connection density, edge cut, or a metric distance between nodes. Generally, the algorithms aim to optimize an objective function for the entire graph.

Dongen et al. [5] introduced the MCL algorithm that uses expansion and inflation operators to characterize the random walk process on the graph and find protein families. It is shown in [6] that this algorithm is one of the best methods to cluster a graph. King et al. in [7] proposed the RNCS algorithm which apportions vertices of a graph using a cost function and a local search algorithm. In another research, Dunn et al. [8] used the Newman average edge algorithm to extract protein complexes. In this algorithm, the edges with the highest average value are removed iteratively until the desired clusters are achieved.

Bader et al. [9] proposed the MCODE algorithm which is one of the first and the most well-known algorithm for predicting protein complexes. In this algorithm, a cluster grows around a seed protein with a greedy approach. The algorithm is composed of three steps: weighting the vertices, predicting the clusters, and preprocessing to filter or add a vertex to clusters.

Jiang et al. [10] presented the SPICi algorithm which was proposed to cluster large biological networks. The clustering process is done by the formation of an initial seed pair and then adding nodes to the initial pair.

Amin et al. [11] introduced the CPA algorithm which uses degree and distance length between two nodes in a cluster. A cluster is made and then removed from the network, and it is repeated until there is no edge left. To form a cluster, the node with the highest weight is chosen as the seed. Then, neighboring nodes are searched and added to the seed through the function. If the function value is the same for multiple nodes, the node with higher distance length is added to the current cluster, and if distance lengths are the same as well, all the nodes will be added to the cluster.

Nepuse et al. [12] proposed the ClusterOne algorithm which takes advantages of a greedy approach. Generally, a seed vertex with the highest degree in the network is chosen. Next, some vertices are added to or removed from the seed through a function with a greedy approach. To detect the overlapping in clusters, all the pair complexes at which the value of a function named Overlap Score exceeds a certain threshold are combined. Finally, the complexes with less than three proteins or density lower than a certain threshold are removed.

Adamcsek et al. [13] proposed the CFinder algorithm. This algorithm is based on the concept of the clique. A cluster can be considered as a small fully connected unit. A 
parameter is used to determine the minimum number of common proteins. First, the algorithm extracts all the subgraphs of the entire network. Second, a matrix is formed to extract the number of common nodes between two cliques. Finally, the cliques with $(k-1)$ common nodes are considered as adjacent and the two cliques are combined.

Pizzuti et al. [14] proposed an algorithm where the operators presented in the restricted neighborhood search clustering (RNSC) algorithm are modified. Instead of evaluating the cost function at each node, a set of nodes is used and the cost function is rewritten. The results showed that even though fewer clusters are predicted in compared with the RNSC method, but high quality clusters were obtained.

Cao et al. [15] presented the MOEPGA algorithm based on a multiobjective function. The main principle of the algorithm is to consider multiple topological properties of the network including name, size, density, and distance length. The algorithm initially analyzes an interactive protein network and then formulates the multiobjective function based on topological properties of the network. Three steps are taken in each subgraph creating the initial population, mutation, and selection.

Lei et al. [16] presented a moth-flame optimizationbased protein complex prediction algorithm, called MFOC. In the MFOC, first, a weighted dynamic PPI network by synthesizing topological and biological information is created. Then, a layer-by-layer scheme is utilized to find the cores of protein complexes as the flames and let the moths fly spirally around the flames to form the complexes.

Sikandar et al. [17] presented an algorithm that considers both topological patterns and biological features for clustering a PPI network. In their algorithm, each complex subgraph is modeled by decision tree learners. They used a training set of known complexes to construct decision trees in depth-first and best-first manner using divide and conquer strategy.

$\mathrm{Gu}$ et al. [18] presented a Markov-based clustering algorithm that uses link similarity to identify the overlapping structures. They have shown in their research that their proposed method can find more modules with biological significance in PPI networks.

Ramadan et al. [3] proposed an algorithm in which the network is clustered by a proposed genetic algorithm so that its population is created by random and spectral methods. To enhance clustering quality, genetic operations and objective functions are adopted. Given that clustering aims to find subgraphs with the high intraconnections, the algorithm first uses spectral clustering to find the minimum cut between two subgraphs. The minimum cut of the network is derived by eigenvector and Laplacian and diagonal matrixes.

Table 1 lists some protein networks clustering algorithms.

\section{Proposed Algorithm}

In this section, we present a new PPI network clustering algorithm based on the genetic algorithm. The genetic Algorithm (GA) is a computational model based on
TABLE 1: Summary of some PPI networks clustering algorithms.

\begin{tabular}{lcccc}
\hline Method 1 & Structure 2 & Class 3 & Overlapping 4 & Reference \\
\hline MCODE & Dense & LD & Yes & {$[9]$} \\
SPICi & Dense & LD & Yes & {$[10]$} \\
CPA & Dense & LD & Yes & {$[11]$} \\
ClusterOne & Dense & LD & Yes & {$[12]$} \\
CFINDER & Dense & LD & Yes & {$[13]$} \\
RNSC & Dense & CL & No & {$[6]$} \\
MOEPGA & Dense & PS & Yes & {$[15]$} \\
Ramadan & Dense & PS & Yes & {$[3]$} \\
MCL & Arbitrary & FS & Yes & {$[4]$} \\
DMCL-EHO & Dense & PS & Yes & {$[19]$} \\
K-means & Arbitrary & CL & Yes & {$[20]$} \\
\hline
\end{tabular}

1: method acronym;2: topological structure a method searches for (arbitrary or dense subgraphs); 3 : the class of the method, where LD:local neighborhood density, CL: cost-based local search, FS : flow simulation, and PS: population-based stochastic search if the method generates overlapping clusters; 4: if the method generates overlapping clusters.

biological evolution which has many advantages over traditional optimization methods. GA can adapt to complex optimization problems and thus a wide range of problem can be solved by it. In most cases, each GA starts with a randomly selected initial population which evolves to achieve a certain goal, i.e., optimizing a function through applying genetic operators (crossover and mutation) to the available chromosomes and creates a new population. The new population is reset on the previous one, and the process is repeated until the algorithm satisfies the termination condition.

Considering the adequate performance of evolutionary algorithms in finding a near-optimal solution to NP-complete problems, we employ an evolutionary algorithm (PPIGA) to find the near-optimal solution to the problem of discovering protein complexes in interactive PPI networks. In the following, the operators used in the PPI-GA are described.

Encoding. GA encodes the possible solutions to a specific problem as a simple chromosome. Choosing the right encoding method is crucial for the performance of the genetic algorithm. Weak encoding scheme can lead a genetic algorithm to converge unacceptable results. We present a new encoding method to represent solutions for clustering PPI networks. To this end, each node (i.e., protein) in the PPI networks is assigned a unique numerical identifier. These identifiers determine which position in the encoded string is used to that node. The method encodes a partition of the PPI network as a string such that each string is a permutation of $\mathrm{N}$ integers. Formally, an encoding, $\mathrm{P}$, is defined as follows:

$$
P=c_{1} \quad c_{2} \quad c_{3} \quad c_{4} \quad \ldots \quad c_{N},
$$

where $N$ is the number of proteins in the PPI networks and $c_{i}$ $(1 \leq i \leq N)$ holds a value in range $[1, N]$. Length of each chromosome is equal to a number of proteins, indicated by $N$. Algorithm 1 shows how we can decode a chromosome into a clustering.

For example, consider the following encoding: 
Let $\mathrm{P}$ is a chromosome which holds a permutation of PPI network. node 1 to $\mathrm{N}$

For each element $i$ in the chromosome

If $(\mathrm{P}[\mathrm{i}] \geq i)$ then

Create a new cluster and assign node $i$ to it.

Else

Assign node $i$ to the same cluster as node $\mathrm{P}[\mathrm{i}]$. End.

Algorithm 1: Decoding a chromosome.

$$
P: 9,3,2,1,4,6,10,7,5,8 \text {. }
$$

In the above permutation encoding, the first value in $\mathrm{P}$ is 9 which is greater than 1 ; hence, a new cluster called cluster 1 is created and assigned node 1 to it. The second value in $P$ is 3 which is greater than 2; hence, node 2 is assigned to a new cluster (cluster 2). Node 3 is assigned to the same cluster as node 2 . Nodes 4 and 5 are assigned to the same clusters as nodes 1 and 4, respectively, because the fourth and fifth values in $P$ are 1 and 4 . Figure 1 shows clustering obtained for this encoding.

Algorithm 2 shows the encoding method described in the above.

Input: a PPI network with $\mathrm{N}$ proteins and $\mathrm{K}$ complexes (clusters)

Output: a Chromosome P with $\mathrm{N}$ genes

Begin

Create an empty chromosome $\mathrm{C}$ with $\mathrm{N}$ genes.

For $i=1$ to $\mathrm{k}$

$\mathrm{A}$ is an array containing nodes in cluster \# $i$

$\mathrm{P}$ is the number of nodes in $\mathrm{A}$

Sort $A$, in ascending order

For $j=1$ to $p-1$

Assign $\mathrm{A}(j)$ to $\mathrm{C}(\mathrm{A}(j+1))$

Next $j$

Assign $\mathrm{A}(\mathrm{p})$ to $\mathrm{C}(\mathrm{A}(1))$

Next i

End

By investigating the proposed encoding, it is obvious that for a given PPI network with $n$ nodes, the search space produced by the encoding for the genetic algorithm may include up to $n$ ! chromosomes which is much smaller in comparison with the $n^{n}$ chromosomes produced by the existing value-based encoding (see [3]). This makes (1) solving the problem easier for the GA and (2) converging faster and finding acceptable solutions during earlier generations due to the reduction in the search space.

Fitness Function. Each chromosome in GA represents an individual (solution). The fitness of each chromosome is computed using an objective function. To evaluate the fitness of chromosomes during the evolutionary process of the algorithm, a multiobjective fitness function is proposed. The

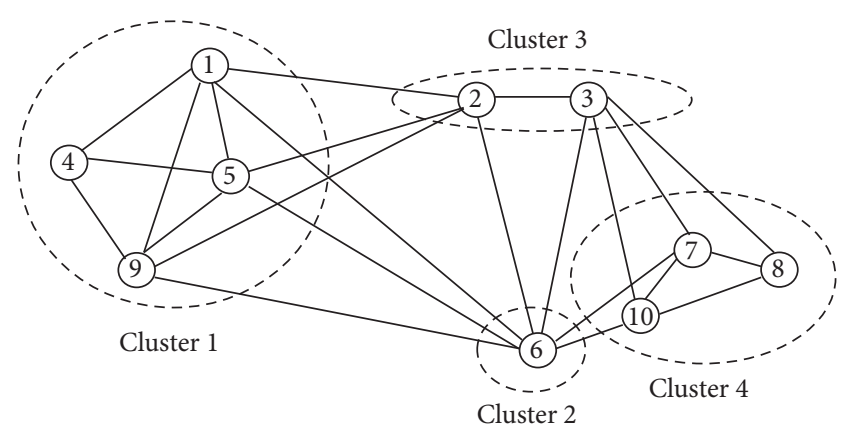

FIgURE 1: A sample clustering.

genetic algorithm aims to optimize this multiobjective function. It is important to note that the output of GA is highly dependent on the fitness function. The first objective function, called CQ, is defined as follows:

$$
\mathrm{CQ}=\sum_{i=1}^{k} C F_{i},
$$

where $C F_{i}$ is calculated as follows:

$$
C F_{i}=\frac{2 \mu_{i}}{2 \mu_{i}+\sum_{j=1}^{k}\left(\varepsilon_{i j}+\varepsilon_{j i}\right)} .
$$

In equation (2), $k$ denotes the total number of clusters, $\mu_{i}$ is the number of edges (i.e., communications) inside the cluster $i$, and $\varepsilon_{i j}$ is the number of edges between cluster $i$ and $j . \mathrm{CF}_{i}$ is defined as a normalized ratio between the total weight of the internal edges and half of the total weight of external edges (edges that exit or enter the cluster). The CQ fitness function calculates communications between the proteins of two different clusters (i.e., inter-relationship) and communications between the proteins of the same cluster (i.e., intrarelationship). The CQ gives higher values while the intrarelationships increase and the interrelationships decrease.

The second objective function is defined as follows:

$$
\text { of }=\sum\left(\mu_{i}-\varepsilon_{i j}\right) \text {. }
$$

This function measures the difference between the number of internal communications of a cluster and the total external communication of that cluster. The larger the number, the better the cluster. Finally, the multiobjective function is introduced as (4): 


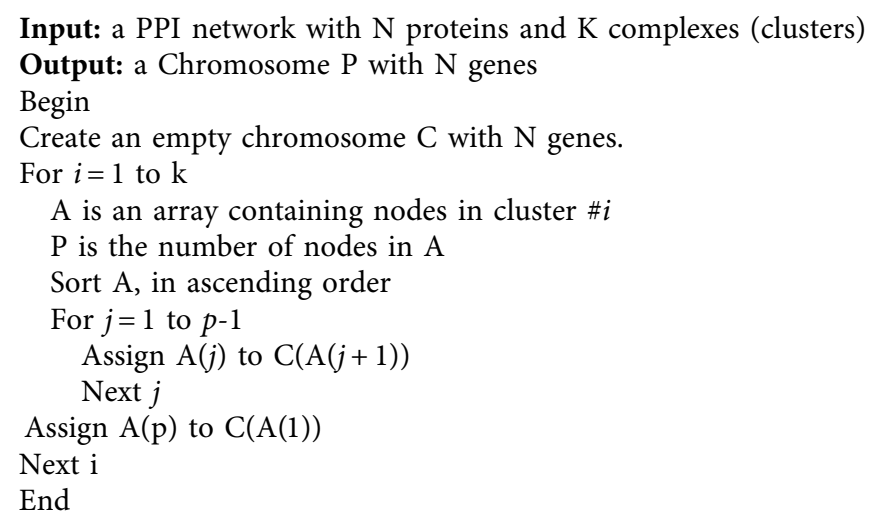

Algorithm 2: Encoding method.

$$
\mathrm{Of}=\mathrm{CQ}+\text { of }
$$

The aim of the CQ function is to maximize the number of intracluster edges $\left(\mu_{i}\right)$ and minimize the number of intercluster connections $(\varepsilon)$. However, because the variables of the functions are not normal, it needs to be normalized. The value of properties should be normalized to common domains.

The range of CQ is between 0 and the maximum number of clusters, and the second objective function is between $(-\varepsilon)$ and $(+\varepsilon)$, which is different from the CQ. This difference leads to small values for the first objective function and large values for the second objective function at each chromosome which means that the effect of the first objective function is ignored. After normalization, the multiobjective fitness function is modified as follows:

$$
\text { Of }=\left(\frac{\mathrm{CQ} / K+\left(\operatorname{sum}\left(\mu_{i}\right)-\operatorname{sum}(\varepsilon)\right) / E}{2}\right) \text {. }
$$

In equation (5), $K$ and $E$ denote the total number of clusters and the total number of edges, respectively, and $\operatorname{sum}\left(\mu_{i}\right)=\sum_{i=1}^{k} \mu_{i}$ and $\operatorname{sum}(\varepsilon)=\sum \varepsilon_{i}$.

Algorithm 3 depicts the pseudocode of our algorithm. In each iteration, the evolutionary operators (selection, crossover, and mutation) are applied to the population, respectively, and the population is steered toward the optimal solution.

Input: protein-protein interaction network (PPI)

Output: a clustered PPI

Algorithm steps are as follows:

(1) Generate the initial population

(2) Evaluate the chromosomes fitness by Equation 5

(3) Generate the intermediate population by employing the standard roulette wheel selection strategy for selecting potentially useful chromosomes for recombination

(4) Apply crossover operator on the intermediate

(5) Apply mutation operator on the intermediate generation
(6) Apply elitism operator

(7) Replace the old population with a new population

(8) If the number of iteration is less than that of generation, go to Step 2; otherwise, stop the iteration steps and return the best result

Initial Population and Operators. To initialize the population, chromosomes to the number of the population are randomly created. Each chromosome is a permutation of $\mathrm{N}$ integers with equal probability. To select chromosomes, the tournament selection method is employed. At first, a certain and limited number of chromosomes (tournament size) is randomly selected. Then, the selected chromosomes are compared and the chromosome with the best fitness is chosen.

To make a crossover operator, because the presented encoding method is a permutation, we need a crossover operator that maintains the permutation form in each chromosome during the evolutionary process of the genetic algorithm. In the PPI-GA, a two-point crossover operator is used. Consider the following two parents:

P1: $C_{1} C_{2}, \ldots, C_{k} C_{k+1} C_{k+3} \cdot C_{k+4} C_{L}$

P2: $D_{1} D_{2}, \ldots, D_{k} D_{k+1} D_{k+3} \cdot D_{k+4} D_{L}$

To build two offspring $\mathrm{O} 1$ and $\mathrm{O} 2$, first, the algorithm generates randomly two positions $\mathrm{k} 1$ and $\mathrm{k} 2$. Middle parts of two offspring are built using the elements between two positions $\mathrm{k} 1$ and $\mathrm{k} 2$ of two parents $\mathrm{P} 1$ and P2:

O1: $\ldots C_{k+1} C_{k+2} C_{k+3} \ldots$

O2: ... $D_{k+1} D_{k+2} D_{k+3} \ldots$

To fill the other parts of $\mathrm{O} 1$ and $\mathrm{O} 2$, the following two lists are created:

List for $O 1,2: D_{k+1} \ldots D_{L} D_{1} D_{2} \ldots D_{k}$ and $C_{k+4} \ldots$ $C_{L} C_{1} C_{2} \ldots C_{k}$

Starting from the 1st position in the list, if the element in the 1st position does not exist in the middle part of $O 1$, it will be assigned to $O 1$. Otherwise, it will be assigned to 02 . If the element in the 2 nd position does not exist in the middle part of $O 1$, it will be assigned to 
Input: protein-protein interaction network (PPI)

Output: a clustered PPI

Algorithm steps are as follows:

(1) Generate the initial population

(2) Evaluate the chromosomes fitness by Equation 5

(3) Generate the intermediate population by employing the standard roulette wheel selection strategy for selecting potentially useful chromosomes for recombination

(4) Apply crossover operator on the intermediate

(5) Apply mutation operator on the intermediate generation

(6) Apply elitism operator

(7) Replace the old population with a new population

(8) If the number of iteration is less than that of generation, go to Step 2; otherwise, stop the iteration steps and return the best result

Algorithm 3: The genetic algorithm process.

O1. Otherwise, it will be assigned to $O 2$, and so on. Analogously, offspring $\mathrm{O} 1$ may be generated as follows:

$D_{k+4} \ldots D_{L} C_{k+4} C_{k+2} C_{k+3} D_{1} D_{2} \ldots D_{k}$

As the same way, for parent $\mathrm{O} 2$, we have the following: $C_{k+4} \ldots C_{L} D_{k+1} D_{k+2} D_{k+2} C_{1} C_{2} \ldots C_{k}$

To illustrate, we apply this method on two sample chromosomes. Consider the following two chromosomes.

$$
\begin{aligned}
& C 1: 9,3,2,1,4,6,10,7,5,8, \\
& C 2: 1,5,3,2,9,7,10,8,4,6 .
\end{aligned}
$$

To build two offspring $O 1$ and $O 2$, two positions 4 and 8 are considered on both chromosomes. Middle parts of two offspring are built using the elements between two positions 3 and 5 of two parents $P 1$ and $P 2$ :

$$
\begin{aligned}
& \text { O1: -, -, -, 1, 4, 6, 10,7,-,-, } \\
& \text { O2: -, -, -, 2, 9, 7, 10, 8,-,-. }
\end{aligned}
$$

To fill the other parts of $O 1$ and $O 2$, the remaining numbers of $\mathrm{O} 2$ and $\mathrm{O} 1$ are, respectively, used as circular:

List for $O 1,2$ is as follows: 4, 6, 1, 5, 3, 5, 8, 9, 3, 2 .

To complete $O 1$, the numbers 4,6 , and 1 are assigned to $O 2$ because they are in the middle of $O 1$. The numbers $5,3,8$, 9 , and 2 are assigned to $O 1$. The remaining numbers are assigned to $\mathrm{O} 2$.

After crossover, the mutation operator is applied to the remaining chromosomes. The swap-based mutation is used in our proposed algorithm.

In the following, we compute the time complexity of the algorithm. Let $N, P$, and $G$ represent the number of proteins, population size, and the number of generations, respectively. We have the following:

(1) To generate the population, several chromosomes each with length $N$ is generated in which each chromosome is a permutation of $N$ integers. So, the order of this step is $O(N \times P)=O(N P)$.
(2) To evaluate the chromosome, for each cluster, it is necessary to count the internal and external relationships. Hence, the order of evaluation of a chromosome is $O\left(N^{2}\right)$. Due to the presence of $P$ chromosomes, the overall order of this step is $O\left(N^{2} \times P\right)$.

(3) Selection step with roulette wheel is in order $O(P)$.

(4) The crossover for each pair will be in $O(N)$, and the mutation is a simple swap in order $O(1)$. So, this step for whole population will be in order $O(N \times P)$.

Steps 2-4 will be repeated $G$ times. Hence, the total order is $\mathrm{O}\left(\mathrm{NP}+G N^{2} \times P+\mathrm{GP}+\mathrm{GN} \times P\right)$. In this paper, $P$ is $10 N$. So, the order is $O\left(N^{2}+G N^{3}+G N+G N^{3}\right)=$ $O\left(G N^{3}\right)$.

\section{The Results}

The PPI-GA clustering algorithm is evaluated on an interaction protein network named Collins. This network is extracted from the BioGrid [21] dataset consisting of 1879 vertices and 140849 edges, which is one of the most important and large-scale datasets in protein-protein networks. The Collins network includes 1004 proteins and 8319 interactions among proteins. The average degree on the network is 16.57 (where the degree of a node in the network is equal to the number of edges connected to a vertex), and the density of the network is 0.016 (which is defined as the number of network interactions per total number of possible edges of network). Figure 2 shows the simulation of this network in the Cytoscape software, which is modeled as an interactive graph. Cytoscape is an open-source bioinformatics software platform for visualizing molecular interaction networks.

To compare the proposed algorithm against existing algorithms, we need a gold standard to evaluate clustering algorithms. We use also CYC2008 [22] and MIPS [23] complexes as the reference clusters which are of the most important benchmarks in detecting the complexity of interacting protein networks (Table 2). Also, the cellular component ontology from Gene Ontology (GO) is utilized to evaluate the coherence of the extracted clusters. 


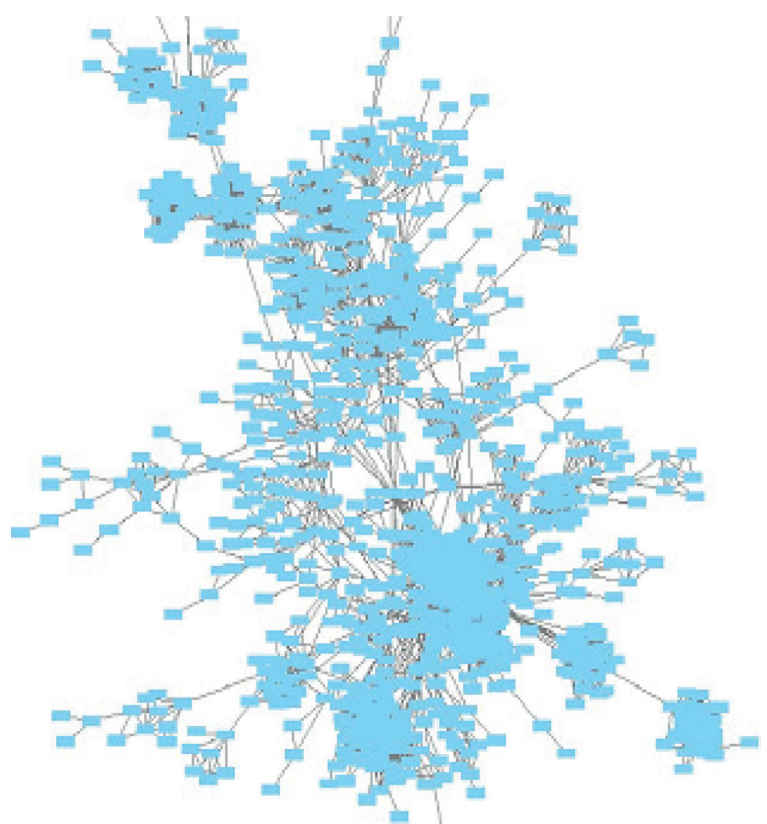

Figure 2: Collins interactive network in Cytoscape application software.

Table 2: Data sets specifications.

\begin{tabular}{lcc}
\hline Benchmark database & \#complexes & \#proteins \\
\hline MIPS & 313 & 1237 \\
CYC2008 & 349 & 1627 \\
\hline
\end{tabular}

4.1. Evaluation. To evaluate the quality of predicted clusters, the Precision (also called positive predictive value), Recall (also known as sensitivity), and F-measure evaluation metrics are employed. These criteria are calculated over pairs of nodes (here proteins). "For each pair of proteins that share at least one cluster in the overlapping clustering results, these measures try to estimate whether the prediction of this pair as being in the same cluster was correct with respect to the underlying true categories in the data (i.e., reference cluster). Precision is calculated as the fraction of pairs correctly put in the same cluster, Recall is the fraction of actual pairs that were identified, and F-measure is the harmonic mean of Precision and Recall" [24]. Let the predicted cluster and the reference cluster be denoted by $C$ and $G$, respectively, and we have

$$
\begin{aligned}
\text { Precision } & =\frac{\mathrm{TP}}{\mathrm{TP} \cup \mathrm{FP}}, \\
\text { Recall } & =\frac{\mathrm{TP}}{\mathrm{TP} \cup \mathrm{TN}}, \\
F-\text { measure } & =2 \times \frac{\text { Precision } \times \text { Recall }}{\text { Precision }+ \text { Recall }}
\end{aligned}
$$

where true positive (TP) is the number of common proteins (vertices) between the predicted cluster $C$ and a cluster belonging to the reference cluster $G$ and true negative (TN) is the number of protein present in the reference cluster $G$ but not found from the predicted cluster $C$. Tables 3 and 4 show clustering results achieved by PPI-GA in 20 independent runs on CYC2008 and MIPS complexes reference, respectively, in terms of Recall, Precision, and F-measure.

These tables show that considering the different metrics, the results obtained by the proposed algorithm in different independent runs are not very different. This shows that the algorithm does not have a random behavior and can always reach an acceptable solution. However, the stability of the proposed algorithm will be examined statistically in the following.

Convergence. During the evolutionary process of algorithm, the population must converge to the optimal solution over generations due to the nature of evolutionary algorithms because these algorithms evolve and improve the entire population.

Figure 3 shows an example of the convergence diagram for the Collins data sample. We carried out the algorithm 20 independent runs for each sample, and we have shown the graph of the best answer here. As it can be seen in the figure, the population of the PPI-GA has consistently converged to the best.

Stability. The stability of an algorithm indicates that the algorithm's performance is correct. The stability of the algorithm means that the results obtained from the algorithm are not scattered so that if the algorithm runs multiple times for the same sample and under the same conditions, the quality of the results in all the performances is at one range with no much difference. To show the stability of the algorithm, we collect the results of 20 independent runs. The results obtained for the Collins data sample in the CYC2008 and MIPS as the reference clusters are shown in Figures 4 and 5 in terms of F-measure, respectively. As seen in the figures, the values obtained are in a range and do not differ greatly, and therefore the solutions obtained from the algorithm are not random and have good stability.

$t$-Test. This test statistically compares the average of two independent samples with a normal distribution. In other words, in this test, the averages gained from random samples are analyzed. This means that we randomly select samples from two different communities and compare the mean of them. This method is based on a normal distribution that is best used when the comparator data have a normal distribution for small samples. In this test, it is assumed that the mean of the data has no significant difference and if the variable value Sig. (2-tailed) is less than the intended error level 0.05 , the hypothesis is rejected and the mean of the data has no significant difference; otherwise, there is not enough reason to reject the hypothesis.

To use this test, the data should have a normal distribution. To prove this, we employ the Kolmogorov-Smirnov test in the IBM statistics SPSS 21 software.

Given the greater amount of Sig. (2-tailed) from 0.05 in all the cases, all of the data used have a normal distribution and $t$-test can be used. The results of the various runs are divided into two groups (first half and the second half). We perform the $t$-test on the samples using the IBM statistical 
TABLE 3: Collins data sample clustering results in 20 independent runs (CYC2008 complex reference).

\begin{tabular}{lcccc}
\hline Run number & \#Clusters & Recall & Precision & F-measure \\
\hline 1 & 69 & 0.878185 & 0.619988 & 0.72683747 \\
2 & 74 & 0.890057 & 0.620371 & 0.731141007 \\
3 & 66 & 0.88961 & 0.602463 & 0.718405897 \\
4 & 71 & 0.89932 & 0.630497 & 0.741289537 \\
5 & 70 & 0.889715 & 0.620035 & 0.730788777 \\
6 & 68 & 0.871675 & 0.609987 & 0.717722023 \\
7 & 73 & 0.880472 & 0.639006 & 0.740552862 \\
8 & 67 & 0.868712 & 0.601529 & 0.710843363 \\
9 & 75 & 0.909976 & 0.611199 & 0.731245808 \\
10 & 72 & 0.875676 & 0.637904 & 0.738113906 \\
11 & 66 & 0.879535 & 0.601817 & 0.714642983 \\
12 & 73 & 0.881598 & 0.630397 & 0.735130386 \\
13 & 71 & 0.894737 & 0.62963 & 0.7391304 \\
14 & 68 & 0.895417 & 0.610328 & 0.725883817 \\
15 & 75 & 0.913626 & 0.623105 & 0.740903813 \\
16 & 72 & 0.889877 & 0.628898 & 0.7369647907 \\
17 & 69 & 0.881598 & 0.617697 & 0.726422348 \\
18 & 74 & 0.900642 & 0.611161 & 0.725199456 \\
19 & 70 & 0.894916 & 0.617349 & 0.73065967 \\
20 & 67 & 0.886518 & 0.602684 & \\
\hline
\end{tabular}

TABLE 4: Collins data sample clustering results in 20 independent runs (MIPS complex reference).

\begin{tabular}{lcccc}
\hline Run number & \#Clusters & Recall & Precision & F-measure \\
\hline 1 & 69 & 0.513214 & 0.350924 & 0.42669397 \\
2 & 74 & 0.498912 & 0.353224 & 0.413614006 \\
3 & 66 & 0.505571 & 0.360712 & 0.421029909 \\
4 & 71 & 0.523451 & 0.345013 & 0.415900716 \\
5 & 70 & 0.490345 & 0.336897 & 0.399389681 \\
6 & 68 & 0.534512 & 0.341284 & 0.416581928 \\
7 & 73 & 0.482342 & 0.345498 & 0.402609674 \\
8 & 67 & 0.509812 & 0.355872 & 0.419154832 \\
9 & 75 & 0.495587 & 0.330721 & 0.396706865 \\
10 & 72 & 0.514067 & 0.347659 & 0.414795446 \\
11 & 66 & 0.523789 & 0.334581 & 0.408331715 \\
12 & 73 & 0.493218 & 0.358721 & 0.415352869 \\
13 & 71 & 0.518116 & 0.363826 & 0.427474985 \\
14 & 68 & 0.509837 & 0.342161 & 0.409499406 \\
15 & 75 & 0.492217 & 0.339464 & 0.401812598 \\
16 & 72 & 0.532116 & 0.348917 & 0.421469612 \\
17 & 69 & 0.517153 & 0.329129 & 0.402253739 \\
18 & 74 & 0.487103 & 0.366129 & 0.418039957 \\
19 & 70 & 0.507113 & 0.348912 & 0.413394072 \\
20 & 67 & 0.528541 & 0.353215 & 0.423447324 \\
\hline
\end{tabular}

software SPSS 21. The results of this test are shown in Tables 5-8. As indicated in the tables, in all the cases, the value Sig. (2-tailed) is more than 0.05 , so there is not enough reason to reject the assumption that the mean of data has no noticeable difference [25].

Therefore, considering Figures 4 and 5 as well as Tables $5-8$, we can claim that the proposed algorithm is stable.

4.2. Comparison. In this subsection, we compare the proposed algorithm to some state-of-the-art algorithms. The setting of parameters is necessary for search-based algorithms. For genetic-based algorithms, for our comparisons, we followed the algorithmic parameters setting. Algorithmic parameters are dependent on the number of proteins.

To set the probability of mutation and crossover operators, twenty different pairs of mutation and crossover probabilities have been tested on the MIPS complex reference using the proposed algorithms. For example, Figure 6 depicts the results.

We obtained the implementations of five of the selected clustering techniques-ClusterONE (https://paccanarolab.org/ cluster-one), MCODE (http://apps.cytoscape.org/apps/mcode), MCL (https://micans.org/mcl/), GA spectral population, and GA random population-from their original authors or official web sites. For the rest of the algorithms, we used the same parameters as those used by the authors of these algorithms. 


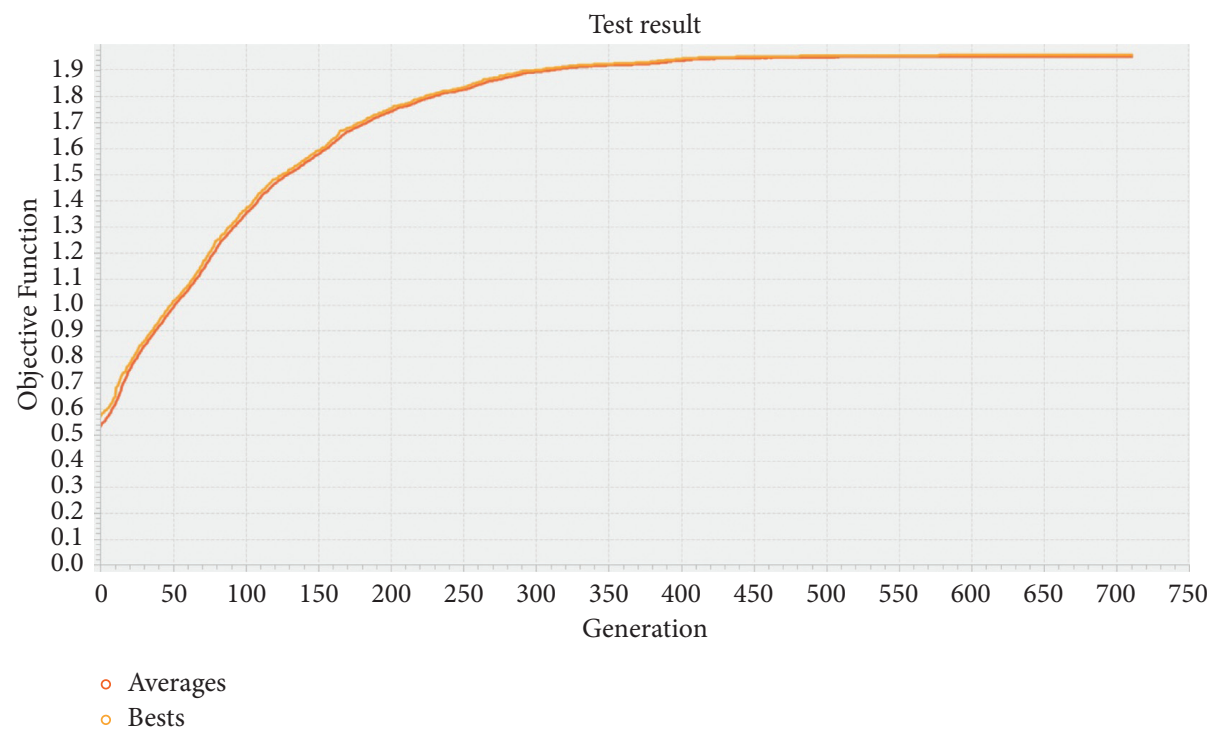

FIGURE 3: Collins convergence diagram of the data sample.

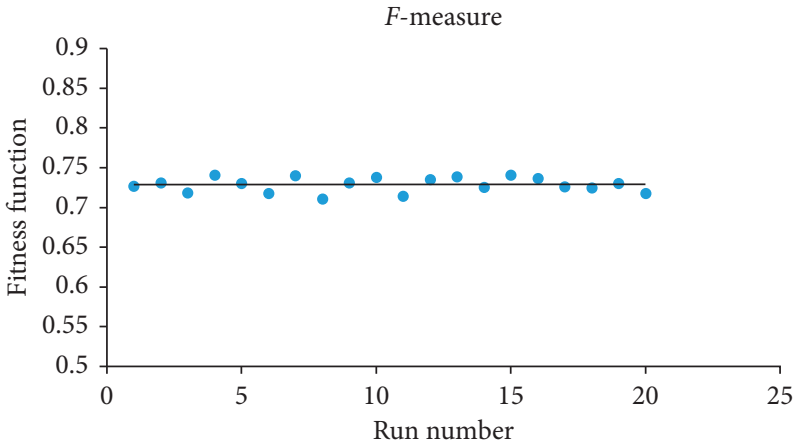

FIgURE 4: The F-measure stability diagram for the CYC2008 data sample.

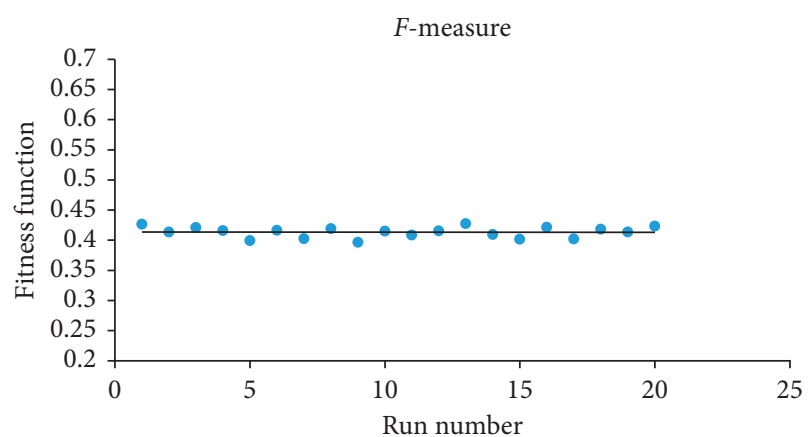

FIgUre 5: The F-measure stability diagram for the MIPS data sample.

Let $n$ denote the number of proteins; the parameter setting for experiments is given in Table 9.

We compared the PPI-GA against DMCL-EHO [19], K-means [20], MCODE [9], MCL [5], ClusterOne [12], and eight algorithms presented in [3] with objectives of Density cut, Maximum cut, Normalized cut, and Ratio cut algorithms on Collins dataset and CYC2008 and MIPS reference
TABLE 5: The Kolmogorov-Smirnov test for the Collins data sample (CYC2008).

\begin{tabular}{lc}
\hline & $F$ measure \\
\hline $\mathrm{N}$ & 20 \\
Normal Parameters & \\
Mean & .7347 \\
Std. Deviation & .01438 \\
Most Extreme Differences & \\
Absolute & .129 \\
Positive & .121 \\
Negative & -.129 \\
Kolmogorov-Smirnov Z & .576 \\
Asymp. Sig. (2-tailed) & .894 \\
\hline a ${ }^{\text {Test distribution is Normal }}{ }^{\text {b }}$ Calculated from data. \\
\end{tabular}

clusters. The results are listed in Table 10. The MCODE algorithm performs clustering based on the density of the protein network and scoring proteins, and it is one of the reference algorithms for detecting the complexes of protein networks. This table shows that the PPI-GA clustering algorithm has an accuracy of 0.63 and 0.51 in clustering the CYC2008 and MIPS references, respectively. These numbers 
TABLE 6: The Kolmogorov-Smirnov test for the Collins data sample (MIPS).

\begin{tabular}{lr}
\hline & VAR00001 \\
\hline $\mathrm{N}$ & 20 \\
Normal Parameters & \\
Mean & .4133776652 \\
Std. Deviation & .0090807204 \\
Most Extreme Differences & \\
Absolute & .151 \\
Positive & .132 \\
Negative & -.151 \\
Kolmogorov-Smirnov Z & .674 \\
Asymp. Sig. (2-tailed) & .754 \\
\hline${ }^{a}$ Test distribution is Normal ${ }^{\text {b }}$ Calculated from data. &
\end{tabular}

TABLE 7: The $t$-test on the Collins sample (CYC2008 reference clusters).

\begin{tabular}{|c|c|c|c|c|c|c|c|c|c|}
\hline \multicolumn{10}{|c|}{ Group Statistics } \\
\hline Number & \multicolumn{3}{|c|}{$\mathrm{N}$} & \multicolumn{2}{|c|}{ Mean } & \multicolumn{2}{|c|}{ Std. Deviation } & \multicolumn{2}{|c|}{ Std. Error Mean } \\
\hline \multicolumn{10}{|l|}{ F-measure } \\
\hline 1 & \multicolumn{3}{|c|}{10} & \multicolumn{2}{|c|}{0.7343} & \multicolumn{2}{|c|}{0.01541} & \multicolumn{2}{|c|}{0.00487} \\
\hline 2 & \multicolumn{3}{|c|}{10} & \multicolumn{2}{|c|}{0.7351} & \multicolumn{2}{|c|}{0.00446} & \multicolumn{2}{|c|}{0.00446} \\
\hline \multicolumn{10}{|c|}{ Independent Samples Test } \\
\hline & \multicolumn{4}{|c|}{$\begin{array}{c}\text { Levene's Test for Equality of } \\
\text { Variances }\end{array}$} & \multicolumn{5}{|c|}{$t$-test for Equality of Means } \\
\hline & \multirow[t]{2}{*}{$\mathrm{F}$} & \multirow[t]{2}{*}{ Sig. } & \multirow[t]{2}{*}{$t$} & \multirow[t]{2}{*}{ dif } & \multirow[t]{2}{*}{$\begin{array}{c}\text { Sig. } \\
\text { (2-tailed) }\end{array}$} & \multirow[t]{2}{*}{$\begin{array}{c}\text { Mean } \\
\text { Difference }\end{array}$} & \multirow[t]{2}{*}{$\begin{array}{l}\text { Std. Error } \\
\text { Differences }\end{array}$} & \multicolumn{2}{|c|}{$\begin{array}{l}\text { 95\% Confidence } \\
\text { Interval of the } \\
\text { Difference }\end{array}$} \\
\hline & & & & & & & & Lower & Upper \\
\hline \multicolumn{10}{|l|}{ F-measure } \\
\hline 1. Equal variances assumed & 0.259 & 0.617 & -0.109 & 18 & 0.915 & -0.00072 & 0.00660 & $-.01459 \mathrm{~s}$ & 0.1316 \\
\hline 2. Equal variances not assumed & & & -0.109 & 17.857 & 0.915 & -0.00072 & 0.00660 & -.01460 & 0.1317 \\
\hline
\end{tabular}

TABLE 8: The $t$-test on the Collins sample (MIPS reference clusters).

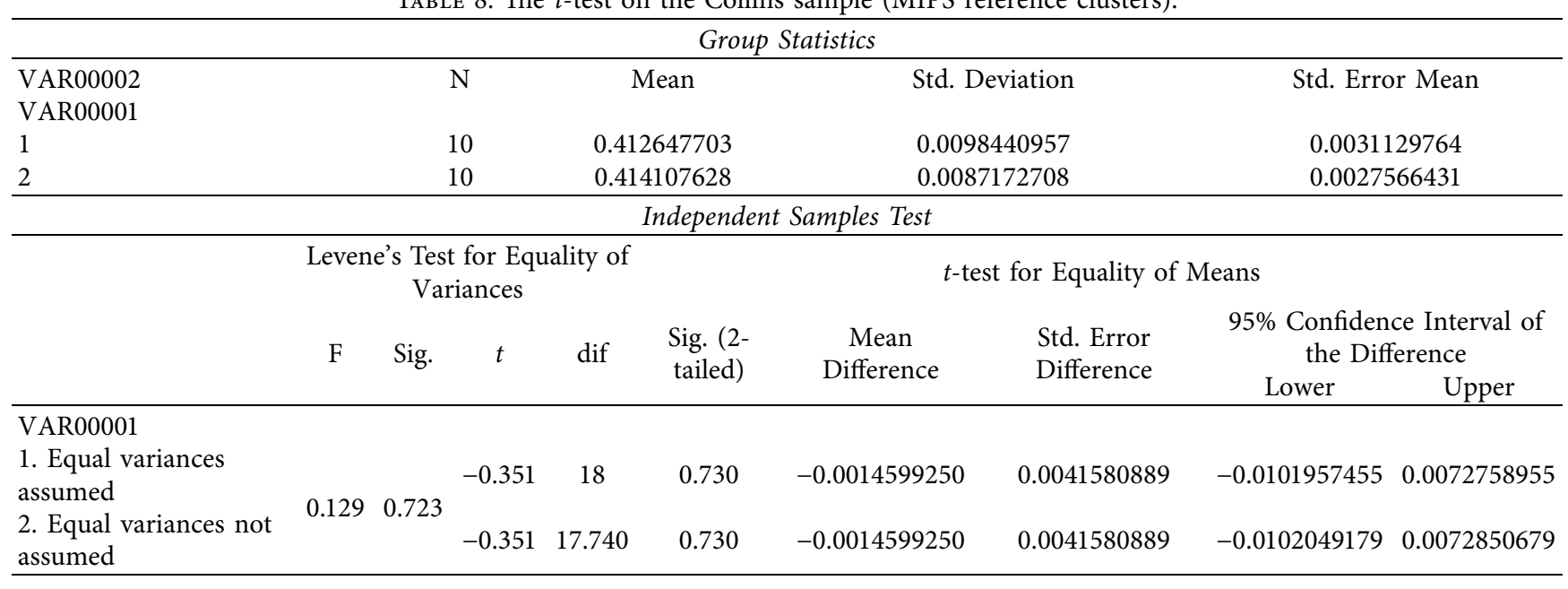

are 0.59 and 0.48 for the MCODE algorithm. For recalling, PPI-GA achieves the value of 0.89 and 0.36 compared with 0.66 and 0.27 for MCODE. The value of $F$-measure for our algorithm is obviously higher than that of the MCODE. Our proposed algorithm shows higher values for Precision, Recall, and F-measure in comparison to MCL which uses random walk process for clustering. For PPI-GA, MCODE, and MCL, the number of predicted clusters is close. Comparing our result with ClusterOne which is based on network density, despite the smaller number of clusters than ClusterOne, PPI-GA presents higher values for Precision, Recall, and F-measure. Ramadan et al. [3] used GA and four different objective functions with the random and spectral initial population for clustering. Comparing the result of PPI-GA with [3], it is shown that PPI-GA can find better solutions in most cases. The value of Precision in [3] is 0.6 and 0.45 . The value of Recall is 0.74 and 0.4 and $F$-measure is 0.66 and 0.41 for CYC2008 and MIPS reference clusters, 


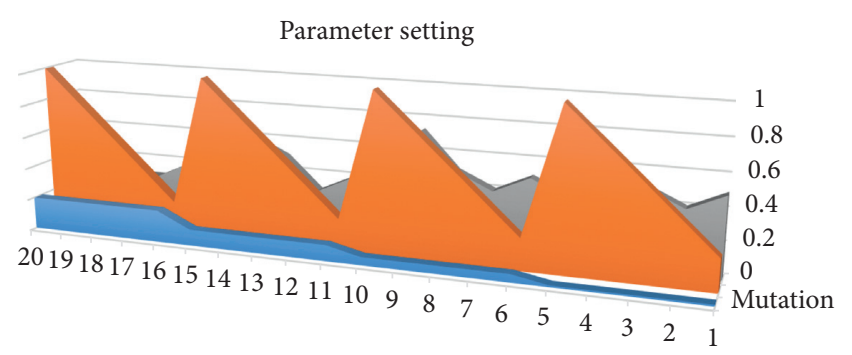

- Mutation

- Crossover

- Recall

Figure 6: Parameter setting for crossover and mutation.

TABLE 9: The parameter setting for experiments.

\begin{tabular}{lc}
\hline Parameter & Values \\
\hline Population size & $10 \mathrm{n}$ \\
Generation & $200 \mathrm{n}$ \\
Crossover rate & 0.8 \\
Mutation rate & 0.05 \\
Selection & Roulette wheel selection \\
Crossover operation & Order \\
Mutation operation & Swap mutation \\
Termination condition & There has been no improvement in the \\
& population for 20 iterations \\
\hline
\end{tabular}

respectively. It is only the Recall value that is higher for [15] than PPI-GA. Recall value is 0.4 and 0.39 for [3] in MIPS in comparison to PPI-GA which provides 0.36 for Recall value. In summary, PPI-GA has the best clustering performance in comparison to other algorithms.

To statistically analyze the results, Cliff's $\delta$ effect size metric is utilized. This test is a nonparametric effect size metric that quantifies the difference among two groups of observations (here, PPI-GA against other tested algorithms). The result of this metric is in range -1 to 1 , and higher value shows that results of the first group (here, PPI-GA) generally are better than those of the second group (other algorithms). To interpret, as [26], the following magnitudes are used: negligible $\quad(|\delta|<0.147)$, small $\quad(|\delta|<0.33)$, medium $(|\delta|<0.474)$, and large $(0.474 \geq|\delta|)$. The results (Table 11) indicate that, in terms of Recall, Precision, and F-measure, the PPI-GA is better than the other algorithms.

Table 12 shows the running time of the proposed algorithm, PPI-GA, against other tested algorithms on MIPS and CYC2008 graphs. We ran the algorithms on a laptop with Intel core i7 processor $2.60 \mathrm{GHz}$ and $16 \mathrm{~GB}$ of memory. On both graphs, the PPI-GA has a shorter running time compared with DMCL-EHO, K-means clustering, Ramadanspectral population-DC (denoted by RS-DC), and Ramadanrandom population-DC (denoted by RR-DC). It is important to note that MCODE, MCL, and ClusterOne use a greedy algorithm to cluster the graphs; hence, they have less execution time than search-based and evolutionary algorithms. $\mathrm{K}$-means can take varying numbers of clusters as input. For $\mathrm{K}$-means, the algorithm has been executed with different values of $k$ in steps of 5 increment, up to a specified runtime.
In terms of the quality of the answers, the proposed algorithm outperforms the algorithms that use greedy methods and is close to them in terms of execution time. As shown in Figure 3 and Table 12, the proposed algorithm has an acceptable speed in reaching an acceptable answer, and this is due to the coding presented in Section 3. The provided coding greatly reduces the search space.

4.3. Clustering Quality. To evaluate the clusters' biological significance in the Collins network, we evaluate the PPI-GA algorithm with components in the Gene Ontology. The GO term finder is applied to obtain the most significant GO terms. The $p$ value is generally applied to measure the probability of enriching a predicted complex via a given GO term by chance. This function is calculated via utilizing the software modules called SGD GO Term Finder (http:// search.cpan.org/dist/GO-TermFinder/) [27]. The $p$ value is defined via the hypergeometric distribution as follows:

$$
p-\text { value }=1-\sum_{i=0}^{k-1} \frac{\left(\begin{array}{c}
M \\
i
\end{array}\right)\left(\begin{array}{c}
N-M \\
n-i
\end{array}\right)}{\left(\begin{array}{c}
N \\
i
\end{array}\right)}
$$

where $N$ is number of proteins in the PPI network, $M$ is size of a list of proteins, $G$ marks to the reference term of interest (the number of proteins in a GO term), $n$ is the number of proteins that are annotated with the same GO term, $k$ is the number of proteins in an extracted cluster $C$, and $i$ is the number of proteins shared between $C$ and $G$. Therefore, the $p$ value closer to zero, the more biologically significant the predicted complexes make. The biological significance of a group is settled by employing a cutoff value to determine significant from insignificant groups. Merely matches with $p$ value below 0.05 are statistically remarkable.

Table 13 collects some of the Collins network' clusters that have a considerable $p$ value. As you can see, the percentages (N\%) are 100 for most clusters, showing that these clusters in the network fit well with the corresponding GO components. Table 13 also clearly demonstrates that genetic algorithm-based methods can identify protein complexes.

The proportion of significant complexes to total predictions is used to evaluate the overall performance of an algorithm. Table 14 shows the comparison results based on this measure. The PPI-GA algorithm has identified 71 protein complexes, of which 67 protein complexes contain gene ontology annotations. In Table 14, the majority of our predicted complexes (94.36\%) are significant and our algorithm also predicts a higher proportion of significant complexes than other algorithms. Meanwhile, Ramadan GA, ClusterOne, and $\mathrm{K}$-means algorithms predict many protein complexes with extremely small size (e.g., with two proteins) and generally predicted complexes with small size tend to have large $p$ value. Therefore, Ramadan GA, ClusterOne, and $\mathrm{K}$-means algorithms only predicted a small proportion of significant complexes. These results are also consistent with the results in Table 11 where these algorithms achieve low 
TABLE 10: Comparison of PPI-GA algorithm results with existing algorithm results.

\begin{tabular}{|c|c|c|c|c|c|c|c|}
\hline \multirow{2}{*}{ Method } & \multirow{2}{*}{ \#Clusters } & \multicolumn{3}{|c|}{ CYC2008 } & \multicolumn{3}{|c|}{ MIPS } \\
\hline & & Recall & Precision & F-measure & Recall & Precision & F-measure \\
\hline PPI-GA & 71 & 0.89 & 0.63 & 0.74 & 0.36 & 0.51 & 0.42 \\
\hline DMCL-EHO & 85 & 0.74 & 0.61 & 0.66 & 0.42 & 0.41 & 0.41 \\
\hline K-means clustering & 120 & 0.55 & 0.67 & 0.60 & 0.28 & 0.40 & 0.32 \\
\hline MCODE & 54 & 0.66 & 0.59 & 0.63 & 0.27 & 0.48 & 0.35 \\
\hline MCL & 75 & 0.65 & 0.45 & 0.54 & 0.27 & 0.34 & 0.30 \\
\hline ClusterOne & 114 & 0.55 & 0.43 & 0.49 & 0.20 & 0.34 & 0.25 \\
\hline \multicolumn{8}{|c|}{ Ramadan GA spectral population ${ }^{1}$} \\
\hline (a) DC & 162 & 0.74 & 0.6 & 0.66 & 0.32 & 0.45 & 0.37 \\
\hline (b) $\mathrm{MC}$ & 180 & 0.71 & 0.47 & 0.6 & 0.38 & 0.40 & 0.39 \\
\hline (c) $\mathrm{NC}$ & 193 & 0.67 & 0.5 & 0.57 & 0.39 & 0.37 & 0.37 \\
\hline (d) RC & 161 & 0.73 & 0.38 & 0.5 & 0.39 & 0.33 & 0.36 \\
\hline \multicolumn{8}{|c|}{ Ramadan GA random population ${ }^{1}$} \\
\hline (a) $\mathrm{DC}$ & 164 & 0.72 & 0.54 & 0.62 & 0.3 & 0.41 & 0.35 \\
\hline (b) $\mathrm{MC}$ & 162 & 0.71 & 0.45 & 0.56 & 0.4 & 0.35 & 0.38 \\
\hline (c) $\mathrm{NC}$ & 138 & 0.66 & 0.57 & 0.61 & 0.36 & 0.44 & 0.41 \\
\hline (d) $\mathrm{RC}$ & 154 & 0.61 & 0.55 & 0.58 & 0.34 & 0.43 & 0.38 \\
\hline
\end{tabular}

${ }^{1}$ DC: Density cut; MC: Maximum cut; NC: Normalized cut; RC: Ratio cut.

TABle 11: Cliff's $\delta$ effect size test.

\begin{tabular}{lccc}
\hline Algorithm & Recall & Precision & F-measure \\
\hline DMCL-EHO & .0 (neg.) & .5 (large) & .5 (large) \\
K-means & .5 (large) & .0 (neg.) & .5 (large) \\
MCODE & .5 (large) & .5 (large) & .5 (large) \\
MCL & .5 (large) & 1 (large) & .5 (large) \\
ClusterOne & .5 (large) & 1 (large) & .5 (large) \\
Ramadan GA spectral-DC & .5 (large) & .5 (large) & .5 (large) \\
Ramadan GA spectral-MC & .0 (neg.) & 1 (large) & .5 (large) \\
Ramadan GA spectral-NC & .0 (neg.) & 1 (large) & .5 (large) \\
Ramadan GA spectral-RC & .0 (neg.) & 1 (large) & .5 (large) \\
Ramadan GA random-DC & .5 (large) & 1 (large) & .5 (large) \\
Ramadan GA random-MC & .0 (neg.) & .5 (large) \\
Ramadan GA random-NC & .25 (small) & .5 (large) \\
Ramadan GA random-RC & .5 (large) & .5 (large) \\
\hline A posive
\end{tabular}

A positive value indicates the effect size favor of the PPI-GA. The interpretation of the effect size is indicated in parenthesis; neg. stands for negligible; med. stands for medium.

TABLE 12: Comparison of average execution time of the proposed algorithm with the existing algorithms in terms of time (minutes).

\begin{tabular}{lcccccccc}
\hline & PPI-GA & DMCL-EHO & K-means Clustering & MCODE & MCL & ClusterOne & RS-DC & RR-DC \\
\hline MIPS & 61 & 68 & $130^{\dagger}$ & 59 & 49 & 53 & 112 \\
CYC2008 & 102 & 123 & $190^{\dagger}$ & 134 & 98 & 110 & 159 & 140 \\
\hline
\end{tabular}

RS-DC and RR-DC indicate Ramadan-spectral population-DC and Ramadan-random population-DC, respectively. $\dagger$ scores in Table 10 show the intermediate clustering results obtained at that time.

scores. In addition, Table 13 shows 14 protein complexes with very low $p$ value, predicted by our PPI-GA algorithm.

\section{Threats to Validity}

In this section, to clarify the validity of PPI-GA, the limitations that can affect the results of the algorithm are discussed. Several factors may bias the validity of the study. These are typically divided into two categories external and internal validity. External validity is about the ability to generalize the results to other than used case studies or indifferent settings for them. (i) The input of the algorithm is a PPI graph, and as other works, in this paper, inter-relationships and intrarelationships are considered as an indicator for PPI clustering. These indicators may not be a good indicator for clustering protein-protein networks or at least may not be sufficient and may require other indicators. However, there is no research that discusses which indicators can improve the quality of PPI clustering.

(ii) In search-based techniques for PPI clustering, generalizing a technique to any protein-protein interaction is an important threat to the validity of 
TABLE 13: Some clusters in the Collins network with the lowest $p$ value with GO component.

\begin{tabular}{|c|c|c|c|c|}
\hline & Size & GO-ID/GO-term & $P$ value & N\% \\
\hline 1 & 17 & "GO:0030880/RNA polymerase complex" & $3.30986 \mathrm{E}-39$ & $100 \%$ \\
\hline 2 & 8 & “GO:0044428/Nuclear part" & $3.70274 \mathrm{E}-05$ & $100 \%$ \\
\hline 3 & 27 & “GO:0005739/Mitochondrion” & $9.82318 \mathrm{E}-22$ & $100 \%$ \\
\hline 4 & 18 & "GO:0000502/Proteasome complex" & $1.76807 \mathrm{E}-40$ & $100 \%$ \\
\hline 5 & 7 & “GO:0030008/TRAPP complex" & 1.02802E-20 & $100 \%$ \\
\hline 6 & 10 & “GO:0044425/Membrane part" & 4.18992E-10 & $100 \%$ \\
\hline 7 & 5 & "GO:0035097/Histone methyl-transferees complex" & $1.31389 \mathrm{E}-11$ & $100 \%$ \\
\hline 8 & 5 & “GO:0030126/COPI vesicle coat" & $1.18247 \mathrm{E}-14$ & $100 \%$ \\
\hline 9 & 15 & "GO:0000502/Proteasome complex" & $2.20275 \mathrm{E}-33$ & $100 \%$ \\
\hline 10 & 12 & “GO:0016514/SWI/SNF complex” & $4.98150 \mathrm{E}-37$ & $100 \%$ \\
\hline 11 & 60 & "GO:0005634/Nucleus" & $2.15384 \mathrm{E}-32$ & $100 \%$ \\
\hline 12 & 81 & "GO:0043227/Membrane-bound organelle" & $4.87516 \mathrm{E}-23$ & $98.4 \%$ \\
\hline 13 & 63 & “GO:0044464/Cell part" & $3.42642 \mathrm{E}-05$ & $95.4 \%$ \\
\hline 14 & 4 & "GO:0031011/INO80 complex" & $4.13601 \mathrm{E}-07$ & $79.0 \%$ \\
\hline
\end{tabular}

TABLE 14: Comparison of various algorithms in terms of significance of the predicted complexes for Collins data.

\begin{tabular}{|c|c|c|c|}
\hline Method & \#Predicted complexes & \#Significant complexes & Proportion (\%) \\
\hline PPI-GA & 71 & 67 & 94.36 \\
\hline DMCL-EHO & 85 & 77 & 90.58 \\
\hline K-means clustering & 120 & 88 & 73.33 \\
\hline MCODE & 54 & 48 & 88.88 \\
\hline MCL & 75 & 37 & 49.33 \\
\hline ClusterOne & 114 & 85 & 74.56 \\
\hline \multicolumn{4}{|c|}{ Ramadan GA spectral population $^{1}$} \\
\hline (a) DC & 162 & 150 & 92.59 \\
\hline (b) MC & 180 & 148 & 82.22 \\
\hline (c) $\mathrm{NC}$ & 193 & 135 & 69.94 \\
\hline (d) $\mathrm{RC}$ & 161 & 110 & 68.32 \\
\hline \multicolumn{4}{|c|}{ Ramadan GA random population ${ }^{1}$} \\
\hline (a) DC & 164 & 143 & 87.19 \\
\hline (b) MC & 162 & 113 & 69.75 \\
\hline (c) $\mathrm{NC}$ & 138 & 124 & 89.85 \\
\hline (d) $\mathrm{RC}$ & 154 & 120 & 77.92 \\
\hline
\end{tabular}

${ }^{1}$ DC : Density cut; MC: Maximum cut; NC: Normalized cut; RC: Ratio cut.

results. So, in this paper, CYC2008 and MIPS datasets, as popular and large datasets, are selected for evaluation and comparison.

Internal validity is concerned with experimental treatments that affect the algorithm results, leading to poor results.

(i) Similar to other encoding methods, duplicate solutions can be generated in the proposed encoding. This may increase the search time in the evolution process of the algorithm. Another threat is that the probability of producing all solutions in the proposed encoding is not the same. This may lead to solutions that are more likely to produce solutions.

(ii) In this paper, metrics such as Precision, Recall, and F-measure are used to compare study results with the current PPI clustering algorithms. Other metrics may produce different results.

(iii) The related rate of crossover and mutation operators used in GA is achieved from several experiments on the CYC2008 and MIPS datasets. However, these numbers may not work well on other datasets.

\section{Conclusion}

Detecting protein complexes is an important analysis method which enables us to understand cellular structures and biological functions within PPI networks. In this paper, we proposed a new approach to detect and predict protein complexes in PPI networks. We proposed a multiobjective function to maximize cluster intraconnections and minimize cluster interconnections. The results showed that the objective function had a better performance compared with other approaches. Our clustering approach is more accurate than MCODE, MCL, ClusterOne, and Ramadan et al.'s algorithms so that it returns better output and higher F-score. In the future, we suggest that the proposed approach can be used for other biological networks such as metabolic networks. The proposed method can be improved for overlapping 
proteins (so that a protein may belong to several clusters). We will try to apply some of the methods presented in [28] to the PPI networks clustering problem and also use other existing methods such as $[29,30]$ instead of genetic algorithms.

\section{Data Availability}

The data used to support this study are available from the corresponding author upon request.

\section{Conflicts of Interest}

The authors declare that they have no conflicts of interest regarding the publication of this paper.

\section{References}

[1] Y. Zhang, H. Lin, Z. Yang, J. Wang, Y. Li, and B. Xu, "Protein complex prediction in large ontology attributed proteinprotein interaction networks," IEEE/ACM Transactions on Computational Biology and Bioinformatics, vol. 10, no. 3, pp. 729-741, 2013.

[2] J. Zahiri, A. Emamjomeh, S. Bagheri et al., "Protein complex prediction: a survey," Genomics, vol. 112, no. 1, pp. 174-183, 2020.

[3] E. Ramadan, A. Naef, and M. Ahmed, "Protein complexes predictions within protein interaction networks using genetic algorithms," BMC Bioinformatics.vol. 17, no. 7, p. 269, 2016.

[4] Z. Wu, Q. Liao, and B. Liu, "A comprehensive review and evaluation of computational methods for identifying protein complexes from protein-protein interaction networks," Briefings in Bioinformatics, vol. 21, no. 5, pp. 1531-1548, 2020.

[5] S. V. Dongen, "A cluster algorithm for graphs," Technical Report INS-R0010, National Research Institute for Mathematics and Computer Science, Amsterdam, The Netherlands., 2000.

[6] S. Brohee and V. Helen, "Evaluation of clustering algorithm for protein-protein interaction networks," BMC Bioinformatics.vol. 7, no. 1, p. 488, 2004.

[7] N. Przulj, D. A. Wigle, and I. Jurisica, "Functional topology in a network of protein interactions," Bioinformatics, vol. 20, no. 3, pp. 340-348, 2004.

[8] R. Dunn, F. Dudbridge, and C. M. Sanderson, "The use of edge between clustering to investigate biological function in protein interaction network," BMC Bioinformatics, vol. 6, no. 1, p. 39, 2005.

[9] G. Bader and C. Hoguean, "Automated method for finding molecular complexes in large protein interaction networks," BMC Bioinformatics.vol. 4, no. 1, p. 2, 2003.

[10] P. Jiang and M. Singh, "SPICi: a fast clustering algorithm for large biological networks," Bioinformatics, vol. 26, no. 8, pp. 1105-1111, 2010.

[11] M. Atlaf-Ui-Amin, Y. Shinbo, K. Mihara, K. Kurokawa, and S. Kanaya, "Development and implementation of an algorithm for detection of protein complexes in large interaction networks," BMC Bioinformatics.vol. 7, no. 1, p. 207, 2006.

[12] T. Nepuse, H. Yu, and A. Paccanaro, "Detection overlapping protein complexes in the protein-protein interaction network," Nature Methods, vol. 9, no. 5, p. 471, 2012.

[13] B. Adamcsek, G. Palla, I. J. Farkas, I. Derényi, and T. Vicsek, "CFinder: locating cliques and overlapping modules in biological networks," Bioinformatics, vol. 22, no. 8, pp. 1021-1023, 2006.

[14] C. Pizzuti and S. E. Rombo, "An evolutionary restricted neighborhood search clustering approach for PPI networks," Neurocomputing, vol. 145, pp. 53-61, 2014.

[15] B. Cao, J. Luo, C. Liang, S. Wang, and D. Song, "MOEPGA: a novel method to detect protein complexes in yeast proteinprotein interaction networks based on MultiObjective Evolutionary Programming Genetic Algorithm," Computational Biology and Chemistry, vol. 58, pp. 173-181, 2015.

[16] X. Lei, M. Fang, and H. Fujita, "Moth-flame optimizationbased algorithm with synthetic dynamic PPI networks for discovering protein complexes," Knowledge-Based Systems, vol. 172, pp. 76-85, 2019.

[17] A. Sikandar, W. Anwar, U. I. Bajwa et al., "Decision tree based approaches for detecting protein complex in protein protein interaction network (PPI) via link and sequence analysis," IEEE Access, vol. 6, pp. 22108-22120, 2018.

[18] L. Gu, Y. Han, C. Wang, W. Chen, J. Jiao, and X. Yuan, "Module overlapping structure detection in PPI using an improved link similarity-based Markov clustering algorithm," Neural Computing and Applications, vol. 31, no. 5, pp. 14811490, 2019.

[19] R. R. Rani, D. Ramyachitra, and A. Brindhadevi, "Detection of dynamic protein complexes through Markov clustering based on elephant herd optimization approach," Scientific Reports, vol. 9, no. 1, pp. 1-18, 2019.

[20] S. Kalaivani, D. Ramyachitra, and P. Manikandan, "K-means clustering: an efficient algorithm for protein complex detection," in Progress in Computing, Analytics and Networking, pp. 449-459, Springer, Singapore, 2018.

[21] T. Reguly, "Comprehensive curation and analysis of global interaction networks in saccharomyces cerevisiae," Journal of Biology, vol. 5, no. 4, p. 11, 2006.

[22] H. W. Mewes, "MIPS: a database for genomes and protein sequences," Nucleic Acids Research, vol. 30, no. 1, pp. 31-34, 2002.

[23] H. W. Mewes, "The bioinformatics of the yeast genome-A historical perspective," Yeast, vol. 36, no. 4, pp. 161-165, 2019.

[24] P. N. Tan, M. Steinbach, and V. Kumar, "Data mining cluster analysis: basic concepts and algorithms," Introduction to Data Mining, vol. 13, pp. 487-533, 2013.

[25] F. A. Paniagua, "The null hypothesis is always rejected with statistical tricks: why do you need it?" Interamerican Journal of Psychology, vol. 53, no. 1, pp. 17-27, 2019.

[26] N. Teymourian, H. Izadkhah, and A. Isazadeh, "A fast clustering algorithm for modularization of large-scale software systems," IEEE Transactions on Software Engineering, p. 1, 2020.

[27] C. Pizzuti and S. E. Rombo, "A coclustering approach for mining large protein-protein interaction networks," IEEE/ ACM Transactions on Computational Biology and Bioinformatics, vol. 9, no. 3, pp. 717-730, May. 2012.

[28] B. Pourasghar, H. Izadkhah, A. Isazadeh, and S. Lotfi, "A graph-based clustering algorithm for software systems modularization," Information and Software Technology, vol. 133, Article ID 106469, 2021.

[29] S. Pourbahrami and S. Azimpour, "A new method for detection of clustering based on four zones Apollonius circle," Iran Journal of Computer Science, vol. 3, no. 1, pp. 59-64, 2020.

[30] M. Bodaghi and K. Samieefar, "Meta-heuristic bus transportation algorithm," Iran Journal of Computer Science, vol. 2, no. 1, pp. 23-32, 2019. 